\title{
A SÍNDROME DE FRAGILIDADE EM IDOSOS: REVISÃO DE LITERATURA SOBRE INSTRUMENTOS DE AVALIAÇÃO E ESCALAS DE CLASSIFICAÇÃ̃O
}

\author{
Mário Pinto \\ Instituto de Ciências Biomédicas Abel Salazar (ICBAS). Porto \\ Portosintesis \\ mppintofp.edu.pt \\ Mariana Pinto \\ Nutricionista unidade hemodialise, Porto \\ Isabel Alçada c Arranhado \\ Universidade Atlântica
}

Recepción Artículo: 8 enero 2021

Admisión Evaluación: 8 enero 2021

Informe Evaluador 1: 10 enero 2021

Informe Evaluador 2: 11 enero 2021

Aprobación Publicación: 12 enero 2021

\begin{abstract}
RESUMO
Antecedentes: 0 perfil de um doente frágil corresponde a uma pessoa idosa, com multimorbilidade, um estado de saúde instável e frequentemente incapacitado. A fragilidade é uma entidade clínica progressiva que pode ser prevenida e tratada, e apresenta-se como uma preocupação e uma prioridade em saúde. Para o seu diagnóstico é essencial ter disponível um instrumento válido, confiável, de fácil aplicação e que possa prever o risco de resultados adversos. Objetivo: 0 presente estudo tem como objetivo central identificar instrumentos especificamente desenvolvidas para avaliar e estratificar a fragilidade entre idosos a partir de informações colhidas pelos profissionais de saúde e identificar aquela que seja mais fácil de usar, segundo critérios estabelecidos pelas Normas de Consensos para a seleção de instrumentos de medida em saúde. (COSMIN) Metodologia: A revisão bibliográfica efetuada no período compreendido entre dia 1 de janeiro de 2013 e 1 de janeiro de 2018. A investigação foi conduzido por dois investigadores, de acordo com os Itens de Relatórios Preferenciais para Revisões Sistemáticas (PRISMA). Foram utilizadas a base de dados da Medline, PubMed, da Embase, da LILACS e da Cochrane Library sem restrição de idioma, definidos e organizados os descritores. Foi usado o PICO (Population Implementation Comparador Outcomes). Foram definidos á priori os critérios a serem seguidos, em relação ao tipo de estudos, medidas de diagnostico, á seleção, tamanho da amostra e á presença de vieses. As principais características de cada instrumento foram estudadas, avaliando sua potencial utilização clínica. Resultados: Dos 881 artigos selecionados, foram identificados 16 estudos com 26 ferramentas de avaliação de fragilida-
\end{abstract}




\section{A SÍNDROME DE FRAGILIDADE EM IDOSOS: REVISÃO DE LITERATURA SOBRE INSTRUMENTOS DE AVALIAÇÃO E ESCALAS DE CLASSIFICAÇ̃̃o}

de. As ferramentas foram classificadas como uni e multidimensional. Os instrumentos unidimensionais são orientados para o domínio físico e da funcionalidade e estado biológico / fisiológico, enquanto as avaliações multidimensionais, baseiam-se na análise das interações dos domínios físico, psicológico e social do funcionamento humano. Foram estudados e comparadas 9 instrumentos, dos quais apenas 3 são clínicos e destes, 2 estratificam a fragilidade. Conclusão: Ainda não existe internacionalmente uma medida padrão e consensual para avaliar a fragilidade; algumas medidas são mais adequadas para rastreio nos hospitais e outras na comunidade. Os domínios que observamos nos instrumentos estudados, são de principalmente uni (físicos/ clínicos) e multidimensional (cognitivo, psicológico, social e ambiental). Dada a complexidade do diagnóstico da fragilidade e do idoso, recomenda-se o uso conjunto de ferramentas físicas e de triagem multidisciplinar.

Palavras-chave: frailty elderly; review; assessments; screening; clinical; tools; scales

\section{ABSTRACT}

Frailty syndrome in the elderly: a review of the literature on assessment tools and rating scales. Background: The profile of a frail patient corresponds to an elderly person, with multimorbidity, an unstable and often disabled health status. Frailty is a progressive clinical entity that can be prevented and treated, and is a health concern and priority. For its diagnosis, it is essential to have available a valid, reliable, easily applicable instrument that can predict the risk of adverse outcomes. Objective: This study aims to identify instruments specifically developed to assess and stratify frailty among the elderly based on information collected by healthcare professionals and to identify the one that is easiest to use, according to criteria established by the Consensus Standards for the selection of measurement instruments in healthcare. (COSMIN) Methodology: A literature review was conducted between January 1, 2013 and January 1, 2018. The research was conducted by two investigators, according to the Preferred Reporting Items for Systematic Reviews (PRISMA). Medline, PubMed, Embase, LILACS and Cochrane Library databases with no language restriction were used, and descriptors were defined and organized. PICO (Population Implementation Comparator Outcomes) was used. We defined a priori the criteria to be followed regarding the type of studies, diagnostic measures, selection, sample size and the presence of biases. The main characteristics of each instrument were studied, evaluating its potential clinical use. Results: From the 881 selected articles, 16 studies with 26 frailty assessment tools were identified. The tools were classified as uniand multidimensional. The uni-dimensional tools are oriented towards the physical domain and functionality and biological/physiological status, while the multidimensional assessments, are based on the analysis of the interactions of physical, psychological and social domains of human functioning. Nine instruments were studied and compared, of which only 3 are clinical and of these, 2 stratify frailty. Conclusion: There is still no internationally agreed upon standard measure to assess frailty; some measures are more suitable for screening in hospitals and others in the community. The domains we observed in the instruments studied, are mainly uni- (physical/ clinical) and multidimensional (cognitive, psychological, social and environmental). Given the complexity of diagnosing frailty and the elderly, the combined use of physical and multidisciplinary screening tools is recommended.

\section{INTRODUÇÃO}

Segundo a Organização Mundial de Saúde (OMS), verifica-se uma acelerada tendência para um progressivo envelhecimento da população mundial, e prevendo-se que nos próximos 50 anos deste século o número de pessoas com mais de 65 anos duplique, podendo atingir em 2050 cerca de 2,1 bilhões de pessoas. A OMS considera também que, "as Doenças não Transmissíveis (DNTs) são res- 
ponsáveis pela morte de 40 milhões de pessoas por ano, o que equivale a 70\% da totalidade de mortes no mundo (1).

A população portuguesa tem vindo a diminuir desde 2010. Atualmente, estima-se um total cerca de 10,3 milhões de habitantes, concentrados nas zonas urbanas e litorais, sendo constituída por uma maioria de idosos e uma minoria de jovens. Verifica-se que $21 \%$ dos portugueses têm 65 ou mais anos e apenas $14 \%$ têm menos de 15 . As pessoas com 75 ou mais anos são cerca de um milhão, sendo a maioria deste grupo etário constituída por mulheres (2).

0 número de pessoas idosas tem aliás vindo a aumentar dramaticamente em quase todos os países, com um consequente aumento da prevalência da fragilidade (3).

0 conceito de fragilidade parece ser consensual entre os investigadores e tem prevalecido 0 contexto clínico (4).Trata-se de um conceito de fragilidade complexo e multifacetado, abrangendo os domínios físico, psicológico e social. A síndrome de fragilidade do idoso é amplamente reconhecida para explicar as variações de riscos para a saúde e para identificar perfis de pessoas idosas tendo em vista a prestação de cuidados personalizados.

Estando o envelhecimento associado o declínio das capacidades físicas e mentais, ao perfil de doente frágil corresponde uma pessoa geralmente idosa, com multimorbilidade, com um estado de saúde instável e frequentemente incapacitada, cujas necessidades de cuidado são determinadas, entre outros fatores, às doenças crónicas, às situações sociofamiliar, económica e ambiental, à acessibilidade a serviços de saúde, à ocupação do tempo livre.

É sabido como as relações sociais desempenham um papel central no bem-estar humano e estão diretamente envolvidas na manutenção da saúde (38). Em contraponto, verifica-se que a solidão se associa com frequência à fragilidade, ao aumento do risco de doenças cardiovasculares, ao aumento da tensão arterial, ao aumento das respostas inflamatórias ao stresse e à mortalidade (5).

A fragilidade é prevalente em idosos, pois atinge apenas 10\% da comunidade em geral (6), mas atinge $80 \%$ de idosos em contexto de cuidados de longa duração (7). Nos hospitais e nos serviços de urgência, entre adultos com mais 85 anos, a prevalência da fragilidade varia entre 50 a $80 \%$ (8) sendo responsável por um peso significativo na procura de cuidados nos serviços de saúde (9).

De acordo com os resultados dos Estudos de Envelhecimento Saudável e Longevidade (D0HEALTH), a prevalência de fragilidade na Europa, variou entre os vários países, tendo a população portuguesa com mais de 70 anos registado o maior grau de prevalência (variação entre $3.1 \%$ e $30.3 \%$ ), e a população austríaca o menor grau (variação entre 0 e $2.5 \%$ )(10).

Os custos dos cuidados prestados a cidadãos com síndrome de fragilidade têm um peso muito significativo no total da despesa dos sistemas de saúde da Europa (11). Conscientes deste facto, os governos têm vindo a desenvolver estratégias para dispensar apoios sociais e serviços de longa duração a pessoas em situação de fragilidade e têm procurado implementar medidas preventivas que visam melhorar a sua qualidade de vida (12).

Tratando-se de uma questão que afeta à escala global um elevadíssimo número de cidadãos e cujas repercussões nos sistemas de saúde se encontram bem e conhecidas, torna-se indispensável dispor de um instrumento que permita identificar a síndrome de fragilidade, em particular entre os cidadãos do grupo etário de idosos, e permita igualmente realizar avaliações seguras conducentes a um encaminhamento adequado.

Verifica-se que atualmente existem mais de 51 instrumentos de medida da fragilidade, subsistindo um amplo debate sobre os mais adequados para utilização na prática clínica. Este debate centrado na procura de um consenso em torno de uma medida padrão que permita o reconhecimento consistente da fragilidade, ocorre um pouco por todo o mundo e é de grande atualidade científica (13). 


\section{A SÍNDROME DE FRAGILIDADE EM IDOSOS: REVISÃO DE LITERATURA SOBRE INSTRUMENTOS DE AVALIAÇÃO E ESCALAS DE CLASSIFICAÇ̃̃o}

0 Royal College of Physicians e a Sociedade Francesa de Geriatria e Gerontologia defendem a triagem da fragilidade em idosos(14) e recomendam algumas ferramentas de diagnóstico da fragilidade física, cognitiva e clínica, habitualmente usadas e validadas, tais como o FRAIL,(15), a Clinical Frailty Scale (16) e o Gérontopôle Frailty Screening Tool (4).

\section{JUSTIFICAÇÃO}

A abordagem científica da fragilidade não tem ainda uma expressão significativa, devendo considerar-se ainda relativamente limitado o conhecimento sobre esta matéria. Por outro lado não se encontra ainda devidamente identificado o modo como os profissionais de saúde, bem como os consumidores dos serviços de saúde, percebem a fragilidade e se é ou não considerada um problema de saúde pública (17).

Considerando-se que os profissionais de saúde - médicos, enfermeiros, nutricionistas, assistentes sociais, psicólogos e outros - têm absoluta necessidade de uma ferramenta de diagnóstico da fragilidade, válida, confiável, fácil e de uso rápido no hospital ou no ambulatório, a identificação de uma dessas ferramentas parece ser muito útil á comunidade.

Verifica-se que existem várias ferramentas de triagem e de diagnóstico de fragilidade na literatura, as quais têm sido usadas em doentes idosos para relacionar a fragilidade com os resultados adversos que dela advêm (18).

\section{OBJETIVOS}

0 presente estudo tem como objetivo central identificar, mediante pesquisa na literatura atual, o conjunto de instrumentos especificamente desenvolvidos para avaliar a existência da fragilidade clínica em pessoas idosas, para proceder à sua análise e eleger aquele que, cumprindo as propriedades clinimétricas e psicométricas requeridas (19), seja mais fácil de usar.

Como objetivos secundários, pretendeu-se: 1ำ comparar o desempenho de diferentes instrumentos de avaliação de fragilidade clínica; $2^{0}$ avaliar sistemática e criticamente a sua confiabilidade, validade e sensibilidade; $3^{\circ}$ avaliar a possibilidade da existência de instrumentos que permitam proceder à estratificação da população frágil; 4ํaำ partir do estudo de cada uma das medidas de fragilidade, identificar possíveis relações entre elas; $5^{0}$ encontrar um instrumento de medida cuja aplicação seja simples, rápida, segura, fiável, reprodutível e não necessite de equipamento especial.

0 conceito de fragilidade e as formas de a medir

Em 2001 Linda Fried e seus colaboradores, nos EUA, propuseram a definição e classificação da fragilidade, através da apresentação do fenótipo de fragilidade com cinco componentes físicos: marcha lenta; autoavaliação de exaustão; perda involuntária de peso no último ano; diminuição da atividade física; diminuição da força e de preensão manuais. Desta definição resultou uma escala de três classes: idosos robustos; pré-frágeis; frágeis (20).

No Canadá, Rockwood e Mitnitski, também no ano de 2001, apresentaram um outro modelo de fragilidade, o modelo baseado em Défices Acumulados, que inclui, não apenas os componentes físicos mas também os aspetos psicossociais da fragilidade (21).

A fragilidade deve ser encarada como uma questão de saúde pública, pelo que a sua prevenção e o seu diagnóstico precoce são fundamentais para a manutenção da qualidade de vida dos mais velhos. A escolha do instrumento de avaliação de fragilidade deve ter em conta o modelo conceptual em que se baseia, a sua forma de administração, a adequabilidade ao contexto e, entre outras propriedades, a sua capacidade preditiva (22).

Naturalmente, os instrumentos destinados a medir a fragilidade devem ser implementadas e avaliadas de acordo com o objetivo do seu uso (23).

Nos últimos anos, fruto da falta de um consenso da definição de fragilidade, assistiu-se ao surgimento de um grande número de instrumentos para avaliação de fragilidade. Tendo em conta o seu 
objetivo, estes instrumentos podem ser agrupados em dois grupos: 1 . 0 grupo clínico/ triagem, definido pela sua dimensão fisiobiológica, portanto unidimensional; 2. 0 grupo multidimensional de rastreio, abrangendo os domínios físico, psicológico e social (24).

A taxonomia e as definições utilizadas para as propriedades clinimétricas das escalas avaliadas seguiram os critérios estabelecidos pelas Normas para Instrumentos de Medida de Saúde (COSMIN) (25) baseadas no COnsensus e foram: Validade, Confiabilidade, Sensibilidade, Especificidade, Valor Preditivo Positivo (VPP), Valor Preditivo Negativo (VPL) e adaptação cultural (26).

\section{METODOLOGIA}

Pesquisa e fontes de informação.

A presente investigação seguiu os princípios de uma revisão de artigos publicados sobre instrumentos de diagnóstico e rastreio de fragilidade, publicados no período de tempo compreendido entre 0 dia 1 de janeiro de 2013 e 1 de janeiro de 2018. Foram definidos os critérios a aplicar: na seleção e análise deste tipo de estudos; na identificação do período de tempo; na seleção e dimensão da amostra; na presença de vieses.

0 objetivo seria encontrar os estudos de revisão sistemática, metanalise, observacionais de coorte com ferramentas disponíveis para diagnóstico clinico e de rastreio, seguindo a metodologia padrão aconselhada para estudos de revisão das guidelines da Cochrane e a lista de verificação PRISMA (27)

Foi usado o motor de busca da Medline, PubMed, da Embase, da LILACS e da Cochrane Library, sem restrição de idioma, usando vocabulário controlado pelo MeSH da MEDLINE(28), para assegurar uniformidade, consistência e precisão. Foram definidos e organizados os seguintes descritores: Frailty elderly, Review, Assessments, Screening, Clinical, Tools, Scales.

Na seleção de artigos e resumos de artigos os critérios de inclusão foram os seguintes: apenas foram considerados artigos de investigação completos e artigos de revisão. Foi igualmente realizada uma "busca lateral", na qual foram pesquisadas as citações de artigos relevantes. Para os identificar, foi usado o Population Implementation Comparator Outcomes (PIC0) (28) (29): População: com idade $\geq 50$ anos. Implementação / indicador, formas de medir a fragilidade em ensaios de controlo observacional, transversal ou randomizado. Comparador: não aplicável e resultados.

Com esta estratégia, houve a garantia da identificação da maioria dos trabalhos publicados, dentro dos critérios estabelecidos. Os artigos selecionados foram incluídos na base de dados Mendeley desktop.

\section{Gráfico 1}

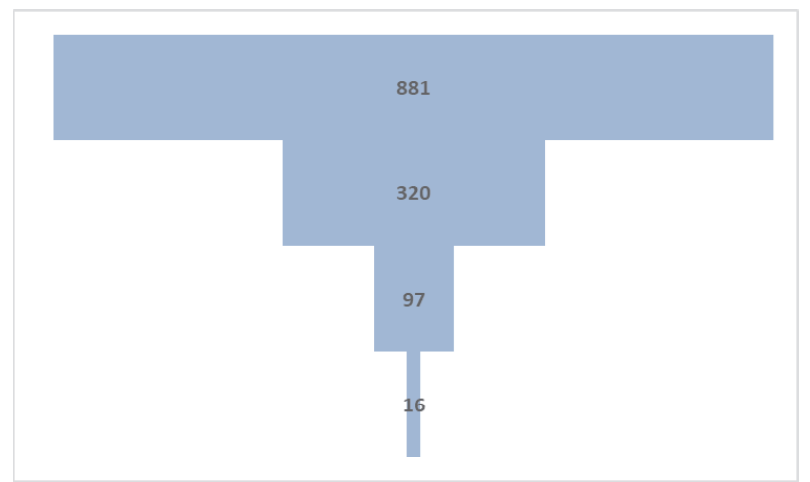

881 Trabalhos iniciais, 320 selecionados,97 elegíveis e 16 para análise 


\section{A SÍNDROME DE FRAGILIDADE EM IDOSOS: REVISÃO DE LITERATURA SOBRE INSTRUMENTOS DE AVALIAÇÃO E ESCALAS DE CLASSIFICAÇ̃̃o}

Dos trabalhos selecionados só 16 cumpriram as condições de inclusão.

Segue-se o diagrama de fluxo de investigação de artigos que identificassem a síndrome de fragilidade e de ferramentas clinicas e de triagem mais usadas.

\section{Quadro 1}

Fases do diagrama do fluxo da pesquisa de artigos sobre ferramentas de identificação de Sindrome de Fragilidade
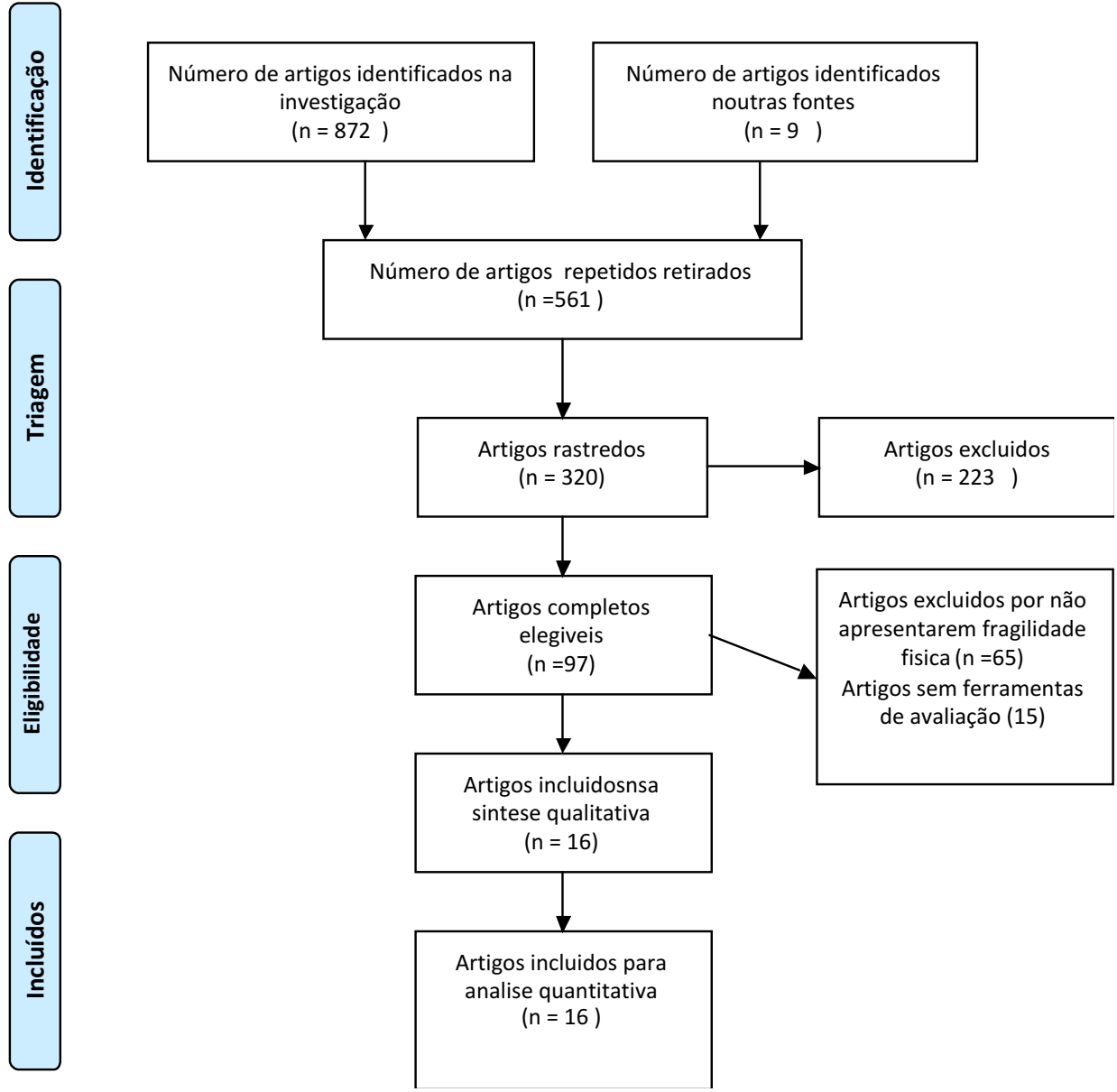

No quadro 1 podemos observar os critérios de elegibilidade de artigos usados para análise

As leituras dos títulos dos artigos e respetivos resumos foi feita por dois investigadores (MP) e (MCP). Na sequência desta leitura efetuou-se uma seleção independente e cega que obedeceu a critérios de inclusão e exclusão previamente definidos: estudos completos, realizados em humanos com idade $\geq$ a 50 anos; que apresentassem escalas de avaliação psicométrica e clinimétricas de fragilidade clínica. 
Foram considerados como critérios de exclusão: estudos em curso, estudos de opinião, cartas ao editor, editoriais, resumos publicados em atas de conferência.

Artigos selecionados na sequência de uma primeira triagem

Foram incluídos alguns estudos que descreviam e testavam a operacionalização dos instrumentos como por exemplo escalas e índices, especificamente desenvolvidos para fazer o rastreio/diagnóstico e triagem da síndrome de fragilidade.

As referências foram geridas recorrendo-se à base de dados do Mendeley e a seleção foi feita pelos dois autores, usando os critérios de elegibilidade acima mencionados.

Sempre que os resumos não se revelaram suficientemente esclarecedores, foi realizada uma leitura na íntegra.

Em seguida, foi feita a leitura completa dos estudos potencialmente interessantes para o presente estudo, da qual decorreu a lista final de inclusão. Quaisquer desacordos entre os autores foram resolvidos recorrendo ao terceiro autor.

Critérios de avaliação dos artigos quanto às escalas de fragilidade.

$\mathrm{Na}$ avaliação dos artigos incluídos na lista resultante da triagem descrita foram analisados os diferentes aspetos, relacionados com as ferramentas usadas para a rastreio e para avaliação clínica da fragilidade: $1^{0}$ tempo necessário para a sua aplicação prática; $2^{\circ}$ potencial uso dos dados obtidos pela avaliação global geriátrica (CGA): $3^{\circ}$ necessidade de utilização de equipamento especializado, por exemplo o dinamómetro manual, avaliação do peso, medição de diâmetros e pregas, testes dinâmicos de levantar e andar, de equilíbrio e marcha; 4Necessidade de treino do utilizador da escala, $5^{\circ}$ avaliação métrica da fiabilidade, da validade, sensibilidade, validade preditiva e estratificação de risco.

Critérios de avaliação da seleção das escalas

Para escolha dos instrumentos de avaliação da fragilidade foram usados os 4 critérios recomendados por Clegg e al: $1^{0}$ inclusão da definição da etiologia biológica, $2^{0}$ inclusão de diagnóstico e planeamento de cuidados, utilizando a avaliação geriátrica integral (AGI); 3ำ possibilidade para medição de outcomes, 4ํestratificação do risco de fragilidade (pré fragilidade), (30). A estes critérios os autores do presente estudo acrescentaram um 5ำ critério: possibilidade de uma utilização simples e rápida por parte dos profissionais de saúde.

Avaliação do Risco de viés entre estudos.

A qualidade dos estudos foi avaliada de forma independente pelos 2 investigadores e pode ser observada no quadro 2.

A avaliação qualitativa dos estudos observacionais e de coorte (9 trabalhos em 16) foi feita recorrendo ao Newcastle Otawa Scale (NOS) que atribui scores expressos em número de estrelas (31) (32). Qualquer discordância na avaliação da qualidade foi resolvida por consenso, resultando uma classificação de cada artigo num mínimo de 7 estrelas, sendo que estudos com scores superiores acima de 7 estrelas são considerados pela NOS com baixo risco de viés.

Nos trabalhos de revisão, a avaliação de qualidade foi confirmada recorrendo à ferramenta AMSTAR(33). Submetidos à cheklist desta ferramenta foram identificados os artigos com uma média de 10 respostas "sim", o que reflete um baixo risco de viés, pois corresponde a 10 pontos em onze possíveis.

Finalmente 0 artigo do ensaio clínico randomizado prospetivo foi avaliado de baixo risco pelo Cochrane Risk Bias(34) 
A SÍNDROME DE FRAGILIDADE EM IDOSOS: REVISÃO DE LITERATURA SOBRE INSTRUMENTOS DE AVALIAÇÃO E ESCALAS DE CLASSIFICAÇ̃̃o

Quadro 2 de viés

\begin{tabular}{|c|c|c|c|c|c|}
\hline $1^{\circ}$ Autor & idades/media & $\begin{array}{l}\text { Viés } \\
\text { NOS }\end{array}$ & $\begin{array}{l}\text { Viés } \\
\text { Amstar }\end{array}$ & Viés CRB & Escalas \\
\hline Rónán O'Caoimh & 80 & 7 & & & 3 \\
\hline $\begin{array}{l}\text { Theodore K. } \\
\text { Malmstrom }\end{array}$ & $>50<65$ & 8 & & & 4 \\
\hline S.J.Wallis & $>75$ & 8 & & & 1 \\
\hline Talal A. & $63( \pm 15)$ & 7 & & & 1 \\
\hline Salina Juna,MD & $>65$ & 7 & & & 1 \\
\hline SJ Moug & $>65$ & & 7 & & 2 \\
\hline Elsa Dent & $>65$ & & 8 & & 15 \\
\hline Bienvenu Bongue & 77,7 & & 8 & & 5 \\
\hline Borja del Pozo-Cruz & $>65$ & 7 & & & 1 \\
\hline Sjors Verlaan MSc & 77,2 & & 11 & & 6 \\
\hline Bem R Davies & $>65$ & & 9 & & 11 \\
\hline Annie Cheung & 76,5 & 8 & & & 2 \\
\hline Edward Chong & 89,4 & 8 & & & 4 \\
\hline Judit Kovacs & $>65$ & & & $\begin{array}{c}\text { Baixo } \\
\text { risco }\end{array}$ & 3 \\
\hline Cecilia G. Ethun & $>65$ & & 10 & & 5 \\
\hline Davide L. Vetrano & $>60$ & & 11 & & 3 \\
\hline
\end{tabular}

Análises adicionais: Sensibilidade e precisão da Investigação

De acordo com o Cochrane Handebook (https://training.cochrane.org/handbook)(27), foi avaliada a sensibilidade e precisão/especificidade(35). A precisão e a sensibilidade nem sempre são reconciliáveis pois uma investigação altamente sensível é frequentemente menos precisa. A sensibilidade é definida como o número de relatórios relevantes identificados, dividindo pelo número total de relatórios relevantes existentes e a precisão e especificidade estabelecem a parte da literatura que deve ser considerada não relevante (negativos ou excluídos). A sensibilidade calculada para os estudos em análise foi de $31 \%$ (97/320) e a precisão e especificidade de $3 \%(223+80 / 881)$, sendo considerada normal entre 2 e $3 \%$. 


\section{RESULTADOS}

Recorrendo ao motor de busca acima referido, foram identificados 872 artigos, aos quais se juntaram 9 disponíveis na biblioteca da Faculdade de Medicina da Universidade do Porto, o que contabilizou um total de 881 artigos.

Foram retirados os artigos duplicados, do que resultou uma seleção de $320(36 \%)$, a submeter à avaliação dos títulos, resumos e conclusões. Deste conjunto foram excluídos 292 por não cumprirem os critérios de elegibilidade, permanecendo para leitura do texto completo 97 artigos, trabaIho que foi realizado pelos 2 investigadores. Após a avaliação do texto completo, foram ainda excluídos 66 estudos por não apresentarem referência à fragilidade física e 15 por não incluírem ferramentas de avaliação (Cf. diagrama anexo).

Finalmente foram admitidos para análise de revisão sistemática 16 estudos publicados entre 2014 e 2018, que abrangeram 440079 participantes e apresentam 26 escalas.

0 Quadro 3 inclui os 16 trabalhos admitidos, apresentando: a respetiva identificação pelo primeiro autor; 0 desenho do estudo; 0 ano em que foi publicado; o país de origem; 0 contexto em que foi desenvolvido; a idade dos participantes ou a sua idade média; o número e tipo de escalas usadas para identificar a fragilidade e se a investigação foi efetuada no hospital ou na comunidade.

Quadro 3. Artigos analisados

\begin{tabular}{|c|c|c|c|c|c|c|c|}
\hline $1^{\circ}$ Autor & Desenho & Ano & País & Amostra & Idades & Escalas & Contexto \\
\hline $\begin{array}{l}\text { Rónán } \\
\text { O'Caoimh }\end{array}$ & Coorte & 2014 & \begin{tabular}{|l} 
Irlanda, \\
Cork
\end{tabular} & 803 & 80 & $\begin{array}{l}\text { CFS, RISC, } \\
\text { Comorbilidades }\end{array}$ & Comunidade \\
\hline S.J.Wallis & Observacional & 2015 & $\begin{array}{l}\text { UK, } \\
\text { Cambridge }\end{array}$ & 11271 & $>75$ & CFS & Hospital/urgência \\
\hline Talal A. & Coorte & 2015 & Canadá & 390 & $63( \pm 15)$ & CFS & Hospital/IR \\
\hline $\begin{array}{l}\text { Salina } \\
\text { Juna,MD }\end{array}$ & \begin{tabular}{|l} 
Coorte \\
Prospetiva
\end{tabular} & 2016 & Canadá & 75 & $>65$ & CFS & Hospital/urgência \\
\hline SJ Moug & Observacional & 2016 & UK Cardiff & 325 & $>65$ & CFS, MoCA & Hospital/urgência \\
\hline Elsa Dent & $\begin{array}{l}\text { Revisão } \\
\text { sistemática }\end{array}$ & 2016 & Austrália & $\begin{array}{l}26 \\
\text { Trabalhos }\end{array}$ & $>65$ & $\begin{array}{l}\text { PFS, FI, SOF, EFS, } \\
\text { FRAIL, CFS, MPI, } \\
\text { TFI, PRISMA 7, } \\
\text { GFI, SPQ, GFS, } \\
\text { Kihon Cheklist, } \\
\text { medidas individuais } \\
\text { de fragilidade }\end{array}$ & Comunidade \\
\hline $\begin{array}{l}\text { Theodore } \\
\mathrm{K} \text {. } \\
\text { Malmstrom }\end{array}$ & $\begin{array}{l}\text { Coorte } \\
\text { Longitudinal }\end{array}$ & 2014 & USA & 998 & $>50<65$ & Frail, SOF, FI, CHS & Comunidade \\
\hline $\begin{array}{l}\text { Bienvenu } \\
\text { Bongue }\end{array}$ & Coorte & 2017 & França & 1643 & 77,7 & $\begin{array}{l}\text { VES 13, CGA, GFI, } \\
\text { PFS }\end{array}$ & Comunidade \\
\hline $\begin{array}{l}\text { Borja del } \\
\text { Pozo-Cruz }\end{array}$ & Transversal & 2017 & Espanha & 519 & $>65$ & Frailty Trait Scale & Comunidade \\
\hline $\begin{array}{l}\text { Sjors } \\
\text { Verlaan } \\
\text { MSc }\end{array}$ & Metanálise & 2017 & Holanda & 5447 & 77,2 & $\begin{array}{l}\text { PFS, SOF, TFI, } \\
\text { EFS, Kihon, CFS }\end{array}$ & Comunidade \\
\hline $\begin{array}{l}\text { Bem R } \\
\text { Davies }\end{array}$ & $\begin{array}{l}\text { Revisão } \\
\text { sistemática }\end{array}$ & 2017 & UK Bristol & 207720 & $>65$ & $\begin{array}{l}\text { eFI, CARS, VES } \\
\text { 13, TFI, GFI, PFS }\end{array}$ & Comunidade \\
\hline $\begin{array}{l}\text { Annie } \\
\text { Cheung }\end{array}$ & $\begin{array}{l}\text { Coorte } \\
\text { retrospetiva }\end{array}$ & 2017 & Canadá & 266 & 76,5 & CFS, FI lab & Hospital/trauma \\
\hline $\begin{array}{l}\text { Edward } \\
\text { Chong }\end{array}$ & $\begin{array}{l}\text { Coorte } \\
\text { prospetiva }\end{array}$ & 2017 & Singapura & 210 & 89,4 & $\begin{array}{l}\text { FI, FRAIL, TFI, } \\
\text { CFS }\end{array}$ & Hospital/Agudos \\
\hline $\begin{array}{l}\text { Judit } \\
\text { Kovacs }\end{array}$ & $\begin{array}{l}\text { Ensaio clínico } \\
\text { prospetivo }\end{array}$ & 2017 & Romania & 57 & $>65$ & $\begin{array}{l}\text { EuroScoreII, CFS, } \\
\text { EFS }\end{array}$ & Hospital/cirurgia \\
\hline
\end{tabular}




\begin{tabular}{|l|l|l|l|l|l|l|l|}
\hline $\begin{array}{l}\text { Cecilia G. } \\
\text { Ethun }\end{array}$ & $\begin{array}{l}\text { Revisão } \\
\text { sistemática }\end{array}$ & 2017 & $\begin{array}{l}\text { USA/ } \\
\text { Atlanta }\end{array}$ & 65436 & $>65$ & $\begin{array}{l}\text { FRAIL, VES 13, } \\
\text { PFS, FI CGA }\end{array}$ & Hospital/Cancro \\
\hline $\begin{array}{l}\text { Davide L. } \\
\text { Vetrano }\end{array}$ & $\begin{array}{l}\text { Revisão } \\
\text { sistemática }\end{array}$ & 2018 & Suécia/Itália & 144403 & $>60$ & CHS, FI, CFS & Hospital/ HTA \\
\hline
\end{tabular}

Abreviaturas: FI-CGA=Frailty Index derived from Comprehensive Geriatric Assessment; EFS = Edmonton Frailty Scale; CFS = Clinical Frailty Scale; CHS=Cardiovascular Health Study Index (PFS=Phenotype Frailty scale); CARS FI-CD=Frailty Index of Accumulated Deficits; GFI;SOF=Study of Osteoporotic Fracture; FRAIL = Fatigue, Resistance, Ambulation, Illness and Loss of Weight Index; Kihon VES 13 MPI = Multidimensional Prognostic Index; TFI = Tilburg Frailty Index, FTS=Frailty Trait Scale, CARS, Fi lab, eFI, EuroScoreI (Surgery scale)

\begin{tabular}{|c|c|c|c|c|c|c|c|}
\hline Escala / Índice & $\begin{array}{l}\text { Tipo de } \\
\text { medida: } \\
\text { clínica/ } \\
\text { rastreio }\end{array}$ & $\begin{array}{l}\text { País } \\
\text { origem }\end{array}$ & $\begin{array}{c}\text { Tempo } \\
\text { (min) }\end{array}$ & Item & Componentes & $\begin{array}{l}\text { Pontos de } \\
\text { corte }\end{array}$ & $\begin{array}{c}\text { Pré } \\
\text { Fragilidade }\end{array}$ \\
\hline FI-CGA & Clínica & Canada & $<15$ & $30+$ & $\begin{array}{c}10 \text { Dominios, } 52 \\
\text { itens, com AVD e } \\
\text { AIVD, } \\
\text { Comorbilidades, } \\
\text { humor e cognição }\end{array}$ & \begin{tabular}{|l} 
Uma escala \\
contínua. \\
Ponto de \\
corte \\
sugerido $>$ \\
0.25 \\
\end{tabular} & Não \\
\hline EFS & Clínica & Canada & $<5$ & 9 & $\begin{array}{c}\text { Cognição, saúde } \\
\text { (2x), internamento, } \\
\text { apoio social, } \\
\text { nutrição, humor, } \\
\text { função, continência }\end{array}$ & $\begin{array}{c}\text { Fragilidade } \\
=\text { pontuação } \\
\geq 7\end{array}$ & Sim \\
\hline CFS & Clínica & Canada & $<5$ & 2 & $\begin{array}{l}\text { Diagrama visual e } \\
\text { escrito da fragilidade } \\
\text { com } 9 \text { imagens } \\
\text { graduadas. } 1= \\
\text { robusto; } 9=\text { doente } \\
\text { terminal e }+3 \\
\text { demência }\end{array}$ & $\begin{array}{c}\text { Uma } \\
\text { pontuação } \\
\text { contínua. } \\
\text { Ponto de } \\
\text { corte Frágil } \\
\geq 5\end{array}$ & Sim \\
\hline CHS-PFS & $\begin{array}{c}\text { Rastreio } \\
\text { e } \\
\text { clínica }\end{array}$ & USA & $<10$ & 5 & $\begin{array}{l}\text { Perda de peso, baixa } \\
\text { atividade física, } \\
\text { exaustão, lentidão, } \\
\text { fraqueza }\end{array}$ & $\begin{array}{l}\text { Fragilidade } \\
\geq 3 \text { itens; } \\
\text { pré-frágeis } 1 \\
-2 \text {; Robusto } \\
=\text { nenhum }\end{array}$ & Sim \\
\hline FI-CD & $\begin{array}{c}\text { Rastreio } \\
\text { e } \\
\text { clínica }\end{array}$ & Canada & $20-30$ & $30+$ & $\begin{array}{c}\text { Défice saúde } \\
\text { acumulados: } \\
\text { pontuação de } 0 \text { (sem } \\
\text { défices) a } 1.0 \text { (todos } \\
\text { os défices) }\end{array}$ & $\begin{array}{c}\text { Escala } \\
\text { Contínua, } \\
\text { ponto de } \\
\text { corte de } \\
\text { fragilidade } \\
\text { sugerido } \\
0,25 \\
\end{array}$ & Não \\
\hline SOF & $\begin{array}{l}\text { Rastreio } \\
\mathrm{e} \\
\text { clínica }\end{array}$ & USA & $<5$ & 3 & $\begin{array}{c}\text { Perda de Peso, } \\
\text { Exaustão, Incapaz de } \\
\text { se levantar da } \\
\text { Cadeira } 5 \text { vezes }\end{array}$ & \begin{tabular}{|l} 
Fragilidade \\
$\geq 2$ itens, \\
por-frágil $=$ \\
1 item; \\
robustos $=0$ \\
itens \\
\end{tabular} & Sim \\
\hline FRAIL & $\begin{array}{c}\text { Rastreio } \\
\mathrm{e} \\
\text { clínica }\end{array}$ & USA & $<10$ & 5 & $\begin{array}{c}\text { Fadiga, Resistência, } \\
\text { deambulação, } \\
\text { Doença, Perda de } \\
\text { Peso }\end{array}$ & \begin{tabular}{|c|} 
Fragilidade \\
$\geq 3$ itens Pré- \\
frágil 1,2 \\
itens; \\
robusto $=0$ \\
item
\end{tabular} & Sim \\
\hline
\end{tabular}




\begin{tabular}{|c|c|c|c|c|c|c|c|}
\hline MPI & $\begin{array}{c}\text { Rastreio } \\
\mathrm{e} \\
\text { clínica }\end{array}$ & Itália & $<15$ & 8 & $\begin{array}{c}\text { Comorbilidades, } \\
\text { Nutrição, Cognição, } \\
\text { Polifarmácia, Risco } \\
\text { de úlcera de Pressão, }\end{array}$ & $\begin{array}{l}\text { Fragilidade> } \\
0,66 \text {; Pré- } \\
\text { fragilidade } \\
=0,34 \text { a }\end{array}$ & Sim \\
\hline & & & & & $\begin{array}{l}\text { Qualidade de Vida, } \\
\text { AVD, AIVD }\end{array}$ & $\begin{array}{c}0,66 ; \\
\text { robusto } \\
<0,34\end{array}$ & \\
\hline TFI & Rastreio & Holanda & $<15$ & 15 & $\begin{array}{c}\text { Autorrelato em } 3 \\
\text { domínios: físico, } \\
\text { psicológico e social }\end{array}$ & $\begin{array}{l}\text { Fragilidade } \\
\text { com Scores }\end{array}$ & Não \\
\hline
\end{tabular}

No que respeita ao desenho, foram encontrados 9 estudos de coorte (56\%), 5 revisões $(31,2 \%)$, um estudo transversal e um ensaio clínico. Quanto ao país de origem dos otrabalhos $9(56,2 \%)$ tem origem na Europa, 5 no continente americano (31,2\%), 1 da Austrália e outro de Ásia (Singapura).

0 conjunto dos trabalhos analisados envolveu mais de 439.563 participantes com idades superiores a 50 anos.

As escalas foram aplicadas em ambiente de prática clínica real, em hospitais, desde 0 serviço de urgência, nos doentes com IR em diálise, em oncologia, nos doentes cirúrgicos, no trauma, durante 0 internamento, nas consultas, nas unidades de medicina, em doentes com IC, hipertensão arterial, nas unidades de longa duração, e na comunidade.

Foram identificados cerca de 26 instrumentos, de entre estes, foram selecionados 9,por serem os mais usados na prática clínica diária e com resultados de validade, fiabilidade, sensibilidade e validade preditiva comprovados e que apresentam componentes clínicos e de rastreio. Destes apenas três apresentam características clínicas e cinco foram classificados como escalas multidimensionais (clínica, psicológico, social e ambiental), isto é, clínicas e de rastreio.

Quanto às escalas selecionadas, cada uma com a sua complexidade, apresentam características de diagnóstico da fragilidade, que permitem facilitar o trabalho dos profissionais, nos hospitais e nos cuidados primários.

Nesta revisão, verificou-se que entre os 16 artigos selecionados a CFS foi a escala mais usada (12 artigos/75\%,), seguindo-se o FI (7 artigos/43,7\%) e o PFS (6 artigos/31\%).

As dimensões das amostras variaram entre um estudo que abrangeu 144403 participantes e outro com apenas 57. A idade destes participantes esteve acima dos 65 anos, exceto num dos estudos selecionados que incluiu idades entre os 50 e os 60 anos. 0 uso das escalas foi mais frequente no ambiente hospitalar (56\%), que na comunidade (43,7\%).

0 quadro 4 apresenta as nove escalas/índices, caracterizadas de acordo com os seguintes elementos: tipo de medida; país de origem; tempo médio gasto na sua aplicação; quantidade de itens usados; número e identificação dos componentes usados; pontos de corte e identificação da pré fragilidade que define o risco e a estratificação da fragilidade.

Este quadro dá conta das características dos instrumentos clínicos e de rastreio mais usadas nos trabalhos selecionados. De acordo com os critérios previamente definidos, procurou-se nos estudos a possibilidade de identificação da pré fragilidade e da estratificação de fragilidade, consideradas indispensáveis no estabelecimento de um plano de intervenção de cuidados adequados a cada pessoa idosa.

As nove escalas analisadas apresentam escalas entre três e nove níveis de fragilidade. Duas dessas escalas não definem a pré fragilidade (TFI e FI-CGA). Os idosos participantes nos estudos foram classificados da seguinte forma: robustos ou não frágeis; pré-frágeis ou vulneráveis; fragilidade 


\section{A SÍNDROME DE FRAGILIDADE EM IDOSOS: REVISÃO DE LITERATURA SOBRE INSTRUMENTOS DE AVALIAÇÃO E ESCALAS DE CLASSIFICAÇ̃̃o}

leve; fragilidade moderada; fragilidade severa. A CFS acrescenta ainda a fragilidade terminal e a fragilidade cognitiva em três níveis. Entre as escalas clínicas mais rápidas, fáceis de usar e com menos itens identificaram-se a EFS e CFS.

0 Quadro 5 mostra-nos os instrumentos de medida clínica com maior número de propriedades clinimétricas foram a Escala Edmonton Frail Scale- (EFS) Clinical Frailty Scale (CFS), não considerando o FI-CGA dado que não define pré fragilidade.

Quadro 5 Medidas Psicométricas e clinimétricas

\begin{tabular}{|c|c|c|c|c|c|c|c|c|}
\hline \multirow[b]{2}{*}{ Escala/Índice } & \multicolumn{7}{|c|}{ Requisitos de medidas de fragilidade } & \multirow[b]{2}{*}{\begin{tabular}{|c} 
Tipo de \\
medida: \\
clinica/rastreio
\end{tabular}} \\
\hline & $\begin{array}{c}\text { Dados } \\
\text { da } \\
\text { CGA }\end{array}$ & $\begin{array}{c}\text { Equipamento } \\
\text { especial }\end{array}$ & $\begin{array}{c}\text { Treino } \\
\text { entrevistador }\end{array}$ & Fiabilidade & Validade & $\begin{array}{l}\text { Validade } \\
\text { Preditiva }\end{array}$ & Sensibilidade & \\
\hline FI-CGA & Sim & Não & Sim & Sim & Sim & Sim & Sim & Clínica \\
\hline EFS & Não & Não & Sim & Sim & Sim & Sim & Sim & Clínica \\
\hline CFS & Sim & Não & Sim & Sim & Sim & Sim & Sim & Clínica \\
\hline CHS & Não & Sim & Não & Sim & Sim & Sim & Não & $\begin{array}{l}\text { Rastreio e } \\
\text { clínica }\end{array}$ \\
\hline FI-CD & Sim & Não & Sim & Sim & Sim & Sim & Sim & $\begin{array}{c}\text { Rastreio e } \\
\text { clínica }\end{array}$ \\
\hline SOF & Não & Não & Não & Não & Sim & Sim & Não & $\begin{array}{l}\text { Rastreio e } \\
\text { clínica }\end{array}$ \\
\hline FRAIL & Sim & Não & Não & Sim & Sim & $\begin{array}{l}\text { Mais } \\
\text { estudos }\end{array}$ & Sim & $\begin{array}{l}\text { Rastreio e } \\
\text { clínica }\end{array}$ \\
\hline MPI & Sim & Não & Sim & Sim & Sim & \begin{tabular}{|c|} 
Mais \\
estudos
\end{tabular} & Mais estudos & $\begin{array}{l}\text { Rastreio e } \\
\text { Clínica }\end{array}$ \\
\hline TFI & Não & Não & Não & Sim & Sim & Sim & Sim & Rastreio \\
\hline
\end{tabular}

0 Tilburg Frailty Index (TFI), sendo uma ferramenta multidisciplinar com componente social, com versões já desenvolvidas em 49 países e adaptação às diferentes línguas e culturas, apresenta todas as características clinimétricas exigidas. Acresce que necessita de mais de 15 minutos para ser aplicados e permite 0 autopreenchimento. No entanto não identifica a pré fragilidade.

\section{DISCUSSÃO}

A análise da literatura realizada pelos 2 investigadores identificou para revisão 26 estudos correspondentes aos critérios definidos. Nestes, encontraram-se nove escalas/ índices, suscetíveis de serem usados na prática clínica diária. No entanto, apenas três foram classificadas como sendo clínicas e seis classificadas como escalas multidisciplinares de triagem.

Da mais de meia centena de instrumentos identificados na literatura que foi objeto da pesquisa para avaliação da síndrome de Fragilidade, verificou-se que nem todas se encontravam bem validadas e só algumas dispunham de validade preditiva.

Atualmente os problemas ligados com o diagnóstico dos níveis de fragilidade são avaliadas com diferentes ferramentas que assume a forma de escalas de avaliação de fragilidade. No entanto a falta de consenso acerca de uma ferramenta padrão para diagnóstico da síndrome de fragilidade, parece ser um problema internacional, ainda não resolvido. 
0 perfil desejado para que uma dessas ferramentas seja padrão, é que cumpra critérios como: ser fácil de usar, ser consensual, que estratifique a fragilidade e tenha provadas com evidencia robusta as propriedades clinimétricas e psicométricas (fiabilidade, validade, de sensibilidade e especificidade).

Nos instrumentos identificados foram observados domínios predominantemente físicos, mas também surgiram igualmente escalas que consideravam fatores de ordem psicológica, social e ambiental. A associação da fragilidade com fatores sociais tem sido amplamente reconhecida, sendo que 0 isolamento social também está significativamente associado à mortalidade.

Um dos pontos de relevância no contexto do diagnóstico da fragilidade é a utilização de abordagens amplas, uma vez que 0 foco exclusivo em problemas físicos pode levar à fragmentação do cuidado ao idoso (36).

Na avaliação e na estratificação da fragilidade nos idosos é, portanto, aconselhável, incluir as diferentes dimensões, nomeadamente as condições sociais e ambientais. No cenário clínico, onde a fragilidade assume importância inquestionável, o desafio atual consiste em operacionalizar o conceito e facilitar seu reconhecimento (4).

Muitos idosos preferem permanecer nas suas próprias casas pelo máximo de tempo possível, em vez de envelhecerem em lares e casas de repouso, devido aos aspetos psicossociais favoráveis de permanecerem no seu ambiente e à possibilidade de gozarem de maior autonomia. Nestes contextos, os instrumentos que identificam a pré-fragilidade ou a fragilidade e os seus fatores de risco devem ser utilizados precocemente, pois a incapacidade resultante da fragilidade pode ser tratada, com um prognóstico mais positivo. Intervenções adequadas e na fase inicial da fragilidade tornamse mais eficazes quando aplicadas em idosos (37)

É ainda necessário considerar que a fragilidade é um processo, caracterizado por frequentes transições ao longo do tempo, sendo a probabilidade de transição para estados de maior fragilidade superior às transições para estados de menor fragilidade e a possibilidade de transição de "muito frágil" para um "estado robusto" extremamente baixa(38).

0 aumento do número de idosos frágeis conduz ao aumento da fragilidade na comunidade. $\mathrm{E}$ uma comunidade frágil gera um aumento do consumo de cuidados e um aumento da despesa pública com dependência de cuidadores. Trata-se de um novo conceito a desenvolver: a de fragilidade das comunidades, decorrente das fragilidades individuais e que carece de identificação e estratificação dos grupos de população frágil.

São vários os cenários onde a avaliação da fragilidade pode ser realizada: nos cuidados de saúde primários; nas unidades de agudos, nos serviços de urgência, nas unidades de trauma, nos hospitais, nas enfermarias, nas consultas de especialidade dos hospitais e ACEs, nas unidades de reabilitação; nas unidades de diálise; nos cuidados ao domicílio, na rede nacional de cuidados continuados; em lares ou casas de repouso; na comunidade; em todos espaços e lugares.

\section{CONCLUSÃO}

A fragilidade é uma condição de saúde heterogenia, progressiva e complexa, que pode ser prevenida e tratada. A sua avaliação precoce e a estratificação de risco para a saúde é a base indispensável para se assegurar a conceção e o desenvolvimento de planos de cuidados dimensionados para as reais necessidades do doente, com o objetivo de reverter 0 seu estadio.

No cenário clínico, a fragilidade assume importância inquestionável, sendo o desafio atual operacionalizar o conceito e facilitar seu reconhecimento usando instrumentos que identificam a préfragilidade. No entanto não existe ainda uma medida internacional padrão e consensual para avaliar a fragilidade. Algumas medidas são mais adequadas para o rastreio da fragilidade ao nível da comunidade e outras para ambiente hospitalar. 


\section{A SÍNDROME DE FRAGILIDADE EM IDOSOS: REVISÃO DE LITERATURA SOBRE INSTRUMENTOS DE AVALIAÇÃO E ESCALAS DE CLASSIFICAÇ̃̃o}

A utilidade de abordagens multidisciplinares reveste-se de grande relevância no contexto da fragilidade, uma vez que o foco exclusivo em problemas físicos pode levar à fragmentação do cuidado ao idoso (36). As relações sociais desempenham um papel central no bem-estar humano e estão diretamente envolvidas na manutenção da saúde (39).

Sendo previsível que, em Portugal, os profissionais de saúde optem preferencialmente por um instrumento adequado ao contexto de seu trabalho que inclua uma escala simples e fácil de aplicar, considera-se que aqueles que oferecem melhores condições são o TFI e a CFS, os quais deverão com vantagens ser traduzidos e adaptados á população portuguesa e usados na população portuguesa.

\section{Recomendações.}

Para o futuro da prestação de cuidados de saúde individualizados e complexos ao idoso será indispensável a avaliação clínica da Síndrome de Fragilidade. Para tal recomendam-se tomar uma de três decisões alternativas:

1- - Criar um consenso generalizado, para disponibilizar, de entre os instrumentos existentes, aquela que seja considerada padrão, não só com o objetivo da investigação, como da prática clinica diária, e que irá permitir estudos comparativos internacionais de prevalência de fragilidade e do seu atendimento.

$2^{\text {a }}$ - Desenvolver um novo instrumento de medida padrão, que tenha por base uma operacionalização consensual do conceito e se associe a uma escala universalmente aceite.

3 a Enquanto não se alcança o desejado consenso, e tendo em conta a complexidade do doente idoso e do diagnóstico de fragilidade, recomenda-se o recurso a uma ferramenta de diagnóstico clinico e a uma outra de triagem multidisciplinar, sendo possível realizar esses procedimentos em duas etapas: primeira etapa -triagem multidimensional, para todos os indivíduos idosos; segunda etapa avaliação apenas para os frágeis) (23).

Agradecimentos

Agradecemos a todos os intervenientes nomeadamente ao Dr. Hélder Reis e à Prof ${ }^{a}$ Dra. Susana Arranhado.

Conflito de Interesses.

Não se identificaram conflito de interesses

\section{REFERENCIAS BIBLIOGRÁFICAS}

1. Direção Geral de Saúde. Depressão e outras Perturbações Mentais Comuns. Direção-Geral da Saúde. 2017;1-104.

2. Turismo TDO, Demogr S, Regi NAS, Portuguesas ES, Territorial R, Territorial R, et al. Retrato Territorial de Portugal Edição 2017. 2017;1-18.

3. Morley JE, Vellas B, Abellan van Kan G, Anker SD, Bauer JM, Bernabei R, et al. Frailty consensus: A call to action. J Am Med Dir Assoc [Internet]. 2013;14(6):392-7. Available from: http://dx.doi.org/10.1016/j.jamda.2013.03.022

4. Morley JE, Vellas B, Kan GA van, al. et. Frailty consensus: A call to action. J Am Med Dir Assoc. 2013;14:392-7.

5. Gale CR, Westbury L, Cooper C. Social isolation and loneliness as risk factors for the progression of frailty: The English Longitudinal Study of Ageing. Age Ageing. 2018;47(3):392-7.

6. Fried LP, Tangen CM, Walston J, Newman AB, Hirsch C, Gottdiener J et al, Fried LP, Tangen CM, Walston $\mathrm{J}$ et al. Frailty in older adults: evidence for a phenotype. J Gerontol A Biol Sci Med Sci. 2001;56(3):M146-56.

7. Topinková E. Aging, disability and frailty. Ann Nutr Metab. 2008;52(SUPPL. 1):6-11. 
8. Shamliyan T, Talley KMC, Ramakrishnan R, Kane RL. Association of frailty with survival: A systematic literature review. Ageing Research Reviews. 2013.

9. Rochat S, Cumming RG, Blyth F, Creasey H, Handelsman D, Le Couteur DG, et al. Frailty and use of health and community services by community-dwelling older men: The Concord Health And Ageing in Men Project. Age Ageing. 2010;39(2):228-33.

10. M.Gagesch, H-A.Bischoff-Ferrari BV. Head-to-head compariso of Fralty Prevalence by instroment in 5 DO-Heath countries,. 2018;1(July):3901295.

11. Sirven N, Rapp T. The cost of frailty in France. Eur J Heal Econ [Internet]. 2017 Mar;18(2):243-53. Available from: https://doi.org/10.1007/s10198-016-0772-7

12. Wyrko Z. CME ACUTE MEDICINE Frailty at the front door. Clin Med (Northfield II). 2015;15(4):377-81.

13. Dent $E$, Kowal $P$, Hoogendijk EO. Frailty measurement in research and clinical practice: A review. Eur J Intern Med [Internet]. 2016 Jun 1 [cited 2018 Feb 27];31:3-10. Available from: https://www.sciencedirect.com/science/article/pii/S0953620516300279

14. Rolland Y, Benetos A, Gentric A, Ankri J, Blanchard F, Bonnefoy M, et al. La fragilité de la personne âgée: un consensus bref de la Société française de gériatrie et gérontologie. Geriatr Psychol Neuropsychiatr Vieil. 2011;9(4):387-90.

15. Hyde Z, Flicker L, Almeida OP, Hankey GJ, McCaul KA, Chubb SAP, et al. Low free testosterone predicts frailty in older men: The health in men study. J Clin Endocrinol Metab. 2010;95(7):3165-72.

16. Rockwood K, Song X, MacKnight C, al. et. A global clinical measure of fitness and frailty in elderly people. CMAJ. 2005;173:489-95.

17. Archibald MM, Ambagtsheer R, Beilby J, Chehade MJ, Gill TK, Visvanathan R, et al. Perspectives of Frailty and Frailty Screening: Protocol for a Collaborative Knowledge Translation Approach and Qualitative Study of Stakeholder Understandings and Experiences. BMC Geriatr. 2017;

18. Huisingh-Scheetz M, Walston J. How should older adults with cancer be evaluated for frailty? Journal of Geriatric Oncology. 2017.

19. Sutton JL, Gould RL, Daley S, Coulson MC, Ward E V., Butler AM, et al. Psychometric properties of multicomponent tools designed to assess frailty in older adults: A systematic review. BMC Geriatr. 2016;16(1).

20. Fried LP, Tangen CM, Walston J, al. et. Frailty in older adults: Evidence for a phenotype. J Gerontol A Biol Sci Med Sci. 2001;56:M146-56.

21. Mitnitski AB, Mogilner AJ, Rockwood K. Accumulation of deficits as a proxy measure of aging. Sci World J. 2001;1:323-36.

22. Coelho T. Modelo integral de fragilidade do idoso (do constructo à avaliação Tilburg frailty indicator). 2014;118.

23. Rockwood K, Theou 0, Mitnitski A. What are frailty instruments for? Vol. 44, Age and Ageing. 2015.

24. Roppolo M, Mulasso A, Gobbens RJ, Mosso CO, Rabaglietti E. A comparison between uni- and multidimensional frailty measures: Prevalence, functional status, and relationships with disability. Clin Interv Aging. 2015;10.

25. Wei Y, McGrath PJ, Hayden J, Kutcher S. Measurement properties of tools measuring mental health knowledge: A systematic review. BMC Psychiatry. 2016;

26. Mokkink LB, Prinsen CAC, Bouter LM, de Vet HCW, Terwee CB. The COnsensus-based standards for the selection of health measurement INstruments (COSMIN) and how to select an outcome measurement instrument. Brazilian J Phys Ther. 2016;20(2):105-13. 


\section{A SÍNDROME DE FRAGILIDADE EM IDOSOS: REVISÃO DE LITERATURA SOBRE INSTRUMENTOS DE AVALIAÇÃO E ESCALAS DE CLASSIFICAÇ̃̃o}

27. Lefebvre C, Glanville J, Wieland LS, Coles B, Weightman AL. Methodological developments in searching for studies for systematic reviews: past, present and future? Syst Rev [Internet]. 2013;2:78. Available from: https://www.scopus.com/inward/record.uri?eid=2-s2.084888333320\& partnerID=40\&md5=1bfa7344c017a96b16aa52a856b510cc

28. Part 1 - Finding the Best Clinical Evidence. 3(3):10-26.

29. Tricco AC, Lillie E, Zarin W, O'Brien KK, Colquhoun H, Levac D, et al. PRISMA extension for scoping reviews (PRISMA-ScR): Checklist and explanation. Ann Intern Med. 2018;169(7):467-73.

30. Clegg A, Young J, Iliffe S, al. et. Frailty in elderly people. Lancet. 2013;381:752-62.

31. Indicate N. Newcastle-Ottawa Quality Assessment Form for Cohort Studies. :17-8.

32. Donato H, Donato M. Etapas na Condução de uma Revisão Sistemática. Acta Med Port. 2019;32(3):227.

33. Zeng X, Zhang Y, Kwong JSW, Zhang C, Li S, Sun F, et al. The methodological quality assessment tools for preclinical and clinical studies, systematic review and meta-analysis, and clinical practice guideline: A systematic review. J Evid Based Med. 2015;8(1):2-10.

34. Kojima G. Frailty as a predictor of hospitalisation among community-dwelling older people: A systematic review and meta-analysis. Journal of Epidemiology and Community Health. 2016.

35. Green S. Cochrane Handbook for Systematic Cochrane Handbook for Systematic Reviews of. 2008;

36. Gobbens RJ, Assen MA van, Luijkx KG, al. et. The Tilburg Frailty Indicator: Psychometric properties. J Am Med Dir Assoc. 2010;11:344-55.

37. Hardy SE, Dubin JA, Holford TR, Gill TM. Transitions between States of Disability and Independence among Older Persons. 2005;161(6):575-84.

38. Gill TM, Gahbauer EA, Allore HG, Han L. Transitions between frailty states among communityliving older persons. Arch Intern Med. 2006;166:418-23.

39. Steptoe A, Shankar A, Demakakos P, Wardle J. Social isolation, Ioneliness, and all-cause mortality in older men and women. 2013;110(15):5797-801. 